\title{
Addressing the burden of cancer and neurological disorders in East Africa: the Royal College of Physicians Medical Training and Fellowship (METAF) programme
}

\author{
Author: Jennifer Eastin
}

\begin{abstract}
Introduction
The Medical Training and Fellowship (METAF) programme is a 4-year project (2016-2020) that aims to increase the early detection, research and treatment of cancer and neurological disorders in East Africa, especially in communities and areas where access to qualified professionals remains a challenge. Through the delivery of a series of clinical training courses, participating physicians will be better equipped to diagnose and manage their patients with common neurological disorders, undertake acute triage of cancer presentation and manage symptoms of cancer within district/regional hospitals.

The programme is a collaboration between the Royal College of Physicians (RCP; technical partner) and the British Council (programme administrator), and is sponsored by the East African Development Bank (EADB).

The METAF programme and partnership are heavily aligned with the United Nations' 2030 Agenda for Sustainable Development and the associated Sustainable Development Goals, recognising non-communicable diseases as a major challenge for sustainable development and supporting the use of partnerships and collaboration across countries and organisations to tackle these challenges and to meet these goals. ${ }^{1}$
\end{abstract}

\section{Methods}

Following a partnership agreement between the RCP and the British Council, funds were secured from the EADB. Within year 1 (2016), a needs assessment was carried out in Kampala, bringing together medical leaders in oncology and neurology from Kenya, Tanzania, Uganda and Rwanda. During year 1, course conveners were recruited, curricula were developed and four intensive training courses were delivered, with RCP volunteers teaching alongside local faculty.

In year 2 (2017), seven training courses were delivered, including the first round of oncology 'cascaded' courses, facilitated by trainers who participated in the previous oncology Training of Trainers (ToT) workshops, with support from course conveners and RCP faculty. In year 3 (2018), 11 training courses were delivered, including a refresher oncology ToT for trainers to regroup and share experiences from the cascaded courses held, and to discuss

Authors: Royal College of Physicians, London, UK the impact of the programme on the trainer's practice and how to improve future trainings. Quantitative feedback to evaluate learning was gathered in the form of multiple-choice tests at the beginning and end of training, with test scores compared to evaluate the knowledge change. Qualitative feedback was gathered in the form of written evaluations at the end of each course. Course content is continually amended based on countryspecific needs, participant and faculty feedback.

\section{Results and discussion}

Since the launch of the programme in 2016, 22 clinical training activities have been delivered: nine neurology training courses, four oncology ToT workshops and nine oncology cascaded training courses. Within year 3, 244 doctors have completed the clinical training courses, bringing the total of doctors trained from across Kenya, Uganda, Tanzania and Rwanda as part of the METAF programme to 448 .

Participant feedback suggests that over 3,000 clinical staff will benefit from the knowledge gained on the clinical courses through mentoring by course participants at home facilities.

\section{Conclusion}

The first 3 years of the METAF programme registered significant strides in addressing the capacity challenges around oncology and neurology in East Africa. Beneficiaries of the training programme came from a wide geographical spread in each country, to ensure that improved diagnosis and treatment in cancer and neurological disorders could be deployed outside the urban teaching hospitals.

Feedback from course participants, course conveners, local and RCP faculty indicates significant gains in learning and that valuable partnerships have been formed. The unique METAF training model, with curriculum design and delivery teams drawing expertise from both the RCP and East Africa, has guaranteed both world-class approach and content while still ensuring contextual relevance. The ToT and a cascade model of training courses has allowed for rapid dissemination of information, maximising reach and increasing teaching capacity. The methodology may be applicable to similar needs in other low- and middle-income countries.

\section{Reference}

1 United Nations. Sustainable Development Goals Knowledge Platform. New York: United Nations. https://sustainabledevelopment. un.org/sdgs [Accessed 6 February 2019]. 